Biosensors \& Bioelectronics 10 (1995) 141-153

\title{
3D Neuro-electronic interface devices for neuromuscular control: Design studies and realisation steps
}

\author{
Wim L. C. Rutten,* Theo A. Frieswijk, Jos P. A. Smit, Tom H. Rozijn' \\ \& Jan H. Meier ${ }^{2}$
}

Institute for Biomedical Technology (BMTI)/Faculty of Electrical Engineering, University of Twente, PO BOX 217, 7500 AE Enschede, The Netherlands

\begin{abstract}
In order to design the shape and dimensions of new 3D multi-microelectrode information transducers properly, i.e. adapted to the scale of information delivery to and from peripheral nerve fibres, a number of studies were, and still are, being performed on modelling and simulation of electrical volume conduction inside and outside nerves, on animal experiments on stimulation and recording with single wires and linear arrays, and on new technologies for 3D micro-fabrication. This paper presents a selection of the results of these 'Neurotechnology' studies at the University of Twente. The experimental and simulation results apply primarily to the peripheral motor nerves of the rat, but are also of interest for neural interfacing with myelinated nerves in man, as fascicles in man are about the same size as in the rat.
\end{abstract}

Keywords: neurotechnology, intraneural stimulation, intraneural recording, neuro-electronic interfacing, FES, selectivity, neuroprostheses, intrafascicular electrodes, multi electrodes, neuromuscular control.

\section{INTRODUCTION}

\subsection{Selective stimulation}

Selective stimulation for neuromuscular control means that electrical artificial activation of peripheral myelinated motor nerve fibres is selective enough to lead to individual and graded control

* Author to whom all correspondence should be addressed.

${ }^{1}$ Present address: Delft Technical University, Physics Dept., Delft, The Netherlands.

2Present address: University Erlangen-Nurnberg, D-8520 Erlangen, Germany. (large dynamic range) of muscle force. This cannot be attained by stimulation from a distance, for example by skin electrodes, nor by circumfascicular electrodes, although in the latter case some progress will be made in that the category of thick myelinated fibres will be relatively favoured over thin fibres: the diameter selectivity has improved. Nerve trunk regions will also be favoured (Sweeney et al., 1990). Unfortunately, the resulting build-up of force is the reverse of natural recruitment behavior in which recruitment proceeds from low to high forces (thin to thick nerve fibres). By activation/blocking stimuli delivered by multiple-cuff electrodes the order of recruitment can be reversed, but the fibres 
still cannot be chosen at will (Zhou et al., 1987). Consequently, the problem of fatigue also cannot be circumvented properly, for example by alternating single fibre activation pulses (Happak et al., 1989).

The only way to control single fibres separately is to provide each fibre with its own interface to the electronic world, preferably at the site of a node of Ranvier, the 'hot spot' for activation. As the number of motor fibers in an average fascicle will be in the order of one hundred and the architecture of a fascicle is not precisely prescribed by nature, it is evident that the best possible approach at present is to employ the possibilities of microfabrication techniques for the construction of a redundant number of microelectrodes in $3 \mathrm{D}$ arrays.

Several modest attempts have already been made, either by inserting linear arrays of microelectrodes in nerve or by ingrowth of fibres through via holes or slits in 'neural sieve' multielectrodes, the number of contact sites being limited to at most 12 , which is far from adequate (one of the sieves has more than 12 via holes, but fewer than 10 are wired) (Edell, 1986; Rutten et al., 1991a; Kovacs et al., 1992).

However, experiments with linear arrays, although insufficient as regards the number of electrodes, are useful for gaining insight into the best possible shape and dimensions (separation distance between electrodes) of multi-electrodes. For example, selectivity tests in stimulation models and experiments with 1D arrays in earlier studies have led to the optimal electrode distance in the design of future 3D arrays, albeit under certain assumptions for the volume conduction (local approach) and statistical distribution of node positions in the fascicle (homogeneous distribution). For example, the peroneal nerve controls four muscles, the hallucis, peroneal, tibial and edl muscles, by stimulation of $632 \pm 27$ $\alpha$ and $\gamma$ motor fibers (Close, 1967; Boyd \& Davey, 1968; Swett et al., 1986). About 55\% of this number, i.e. 350, are $\alpha$ motor fibres. The corresponding nodal density is $1.8 \times 10^{12}$ nodes/ $\mathrm{m}^{3}$ and electrodes must be separated by $128 \mu \mathrm{m}$ for maximal selectivity (Rutten et al., 1991a, b).

Under the same simple model assumptions, it was also predicted that recruitment curves obey a power law with a power of three (in the low force range), i.e. twitch force varies with stimulus current pulse amplitude as $F \sim P$. Further study, reported in this paper, has shown that this power-law behavior is not often seen. Usually, recruitment curves are steeper. By more careful modelling of the nerve excitation process and accounting for the random distribution of fibers over the fascicle cross section, as well as incorporating a fiber diameter distribution, it appeared possible to bring model simulations of recruitment in much better agreement with experimental results.

\subsection{Selective recording}

The forward control of muscle by artificial stimulation might gain considerable importance when this control is supplemented by selective feedback information from nerve fibers attached to muscle spindles, tendon organs, cutaneous sensors, etc. (Calancie \& Stein, 1988).

Several factors make selective recording less easy to deal with than stimulation.

First, although selective recording at first glance might just seem the reciprocal of selective stimulation, this is not what happens in a nerve. While the point source of an electrode site in stimulation is replaced in recording by a series of about 20 current sources ( 20 active nodes of Ranvier) carrying the signal of an active nerve fiber, the activation of a fiber does not need the disturbance of equilibrium in more than about three nodes.

Second, the literature on micro-neurographic recording with tungsten electrodes in human nerve in general confirms the time course of single fiber action potentials (typically $0.5 \mathrm{~ms}$ ) to be expected from animal electrophysiology, but the shape and amplitude may differ considerably from the standard expectation (biphasic, with a small third phase, amplitudes up to $0.5 \mathrm{mV}$ ) (Brink \& Mackee, 1993).

Both factors ask for specific definitions of selectivity in modelling and experimentation during the search for recordings of single fiber action potentials.

This paper discusses the modelling of extracellular recording in peripheral nerve and presents the consequences for the design of a $3 \mathrm{D}$ multielectrode. It also shows recordings made with linear arrays.

\subsection{D design}

The foregoing implies that much can be learned about the design of an optimally selective multi- 
micro-electrode from experiments with single wire electrodes and linear arrays, as well as by concomitant volume conduction modelling.

The actual design, however, is more than just the dimensions: the 3D construction must be realisable by micro-machining techniques, the electrode material must deliver the right current density and be non-toxic, and must not exceed the reversible charge injection limit (Robblee \& Rose, 1990). Furthermore, the electrodes must be contacted and multiplexed properly, and current sources should be on board, as well as buffer amplifiers, etc. This paper presents an overview of the micro-fabrication process.

\section{METHODS}

\subsection{Animal experiments: stimulation and recording}

Acute experiments were performed on anaesthetized Wistar rats, using the peroneal nerve of the right hindleg, of which the tibial and edl muscles were exposed and connected to force transducers (stimulation experiments) or actuators (recording experiments). Electrical stimuli were $100 \mu \mathrm{s}$ rectangular cathodic pulses, rate $1 \mathrm{~Hz}$, with current amplitudes from 1 to $100 \mu \mathrm{A}$ (step $0.1 \mu \mathrm{A}$ ), in order to elicit twitch force isometrically. In recording, the muscles were passively stretched with short force ramps $(2-10 \mathrm{~ms})$ or steps, up to a force of $2 \mathrm{~N}$. Stimuli in the nerve were applied or action potentials recorded using a 12-fold linear micro-array (Pt electrode sites $10 \times 50 \mu \mathrm{m}$, interdistance $50 \mu \mathrm{m})$, as reported earlier. The array was inserted perpendicularly to the fiber direction (see also Fig. 1).

A number of stimulation experiments were also performed with randomly inserted $25 \mu \mathrm{m}$ diameter, coated $\mathrm{Ni}-\mathrm{Cr}$ wires. The exposed length of peroneal nerve was about $2 \mathrm{~cm}$, which was either immersed in Ringer solution or suspended in air.

Recruitment curves were measured under one of two conditions. Either stimulation current was chosen at a number of values and twitch force was the averaged result of 16-64 twitches at each current, or current was increased in steps of $0.2 \mu \mathrm{A}$ and each twitch response was measured.

Action potentials were recorded bipolarly at pairs 1-2, 3-4, etc., of the 12-fold array. As a check, the compound action potential of the whole nerve was monitored bipolarly, at a position distally from the array, by a double hook wire electrode. Each recording was the averaged result from 16-64 stretch stimuli.

The impedances of the 1D array electrode sites were measured in the range $50 \mathrm{~Hz}-50 \mathrm{kHz}$. At $10 \mathrm{kHz}$, a typical impedance absolute value is $100 \mathrm{k} \Omega \mathrm{hm}$. Care was taken that impedance differences between electrodes in a bipolar pair were negligible compared to the input impedances of amplifiers $(10 \mathrm{M} \Omega \mathrm{hm})$, so that no common mode artefacts were recorded. Recording amplifiers had a passband of $50 \mathrm{~Hz}$ to $10 \mathrm{kHz}$. Signals were sampled with a frequency of 20 or $100 \mathrm{kHz}$.

\subsection{Modelling and simulation}

\subsubsection{Stimulation}

The usual way to model the effect of stimulatory currents from a micro-electrode point source on nerve fibre excitation is a two-step procedure.

First, the response of a nerve fibre to an electrical field is modelled. For this, we use the activating function approach, in which a section of the fibre is modeled by an RC-network. The nerve becomes active when the second-order difference potential $f$ of external node potentials $V_{\mathrm{e}}$ of a node and its two neighbours exceeds a chosen threshold $(20 \mathrm{mV})$. As the exact node positions are unknown and $f$ for a given diameter class of fibres only depends on the internode distance $\lambda$, activating functions are calculated for each position $x, y, z$ and $x, y, z \pm \lambda$ in the fascicle, for each electrode. Thus:

$$
\begin{aligned}
f= & V_{\mathrm{e}, n-1}-2 V_{\mathrm{e}, n}+V_{\mathrm{e}, n+1}=V_{\mathrm{e}}(x, y, z-\lambda) \\
& -2 V_{\mathrm{e}}(x, y, z)+V_{\mathrm{e}}(x, y, z+\lambda)
\end{aligned}
$$

Second, the potential fields generated by electrode currents are calculated at the node positions. The combination of the two determines whether a specific nerve fibre will fire or not. (The simplified procedure, the local approach mentioned in the introduction, is an approximation, in that the electrode is so close to one node that the two neighbours can be neglected; also, the medium is taken as a homogeneous, isotropic conductor.)

Figure 2 shows the volume conduction model. The fascicle is idealised as an electrical homogeneous and infinitely long extending cylinder with a radial conductivity $\sigma_{\mathrm{r}}$ and a longitudinal conductivity $\sigma_{\mathrm{z}}$. The cylinder is surrounded by a layer that represents the thin perineurium, with 


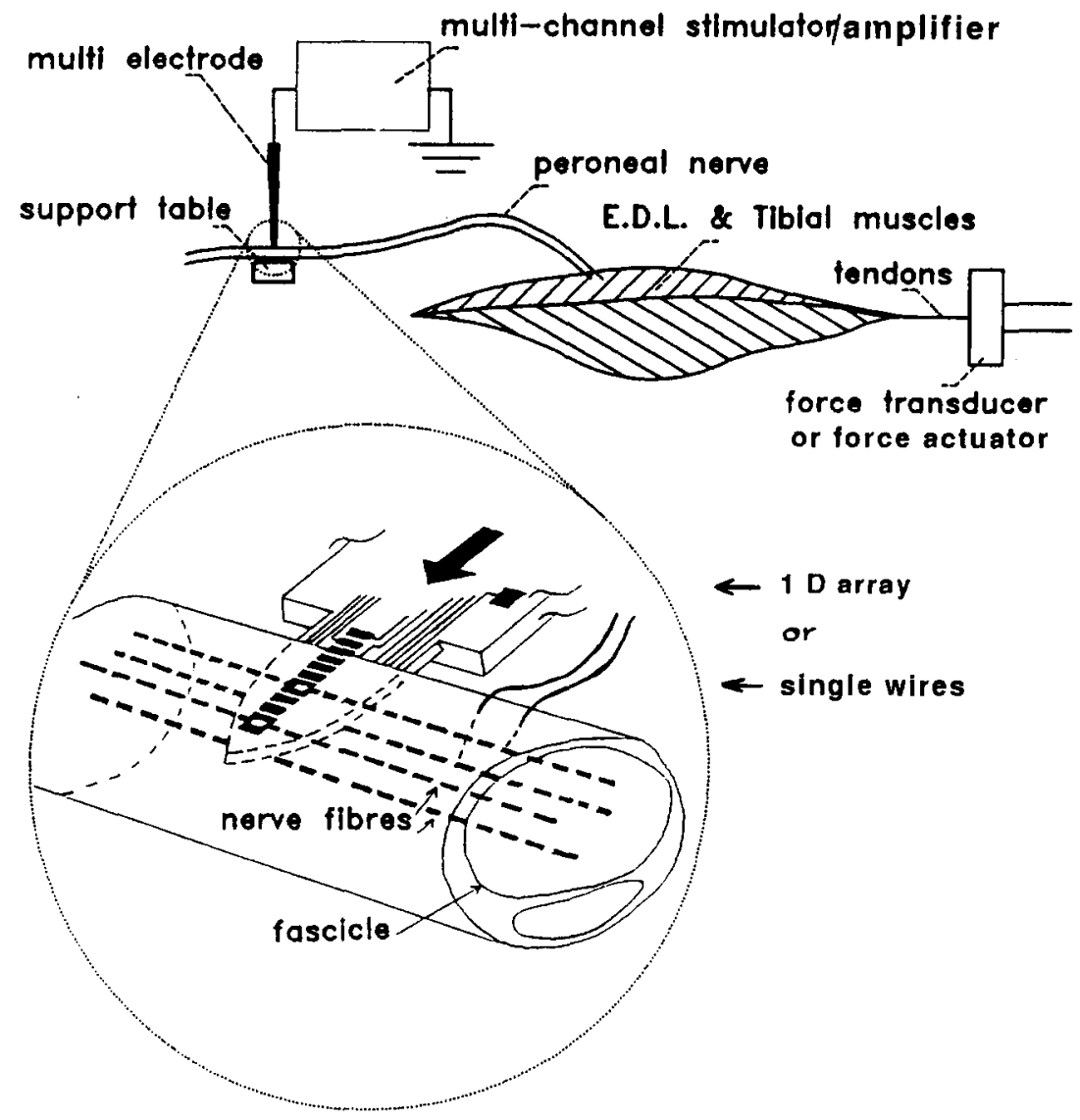

Fig. 1. The linear stimulation array with 12 electrode sites (spacing $50 \mu \mathrm{m}$ ) and the animal experimentation set-up for stimulation and recording. The array is inserted perpendicularly into the peroneal nerve of the rat, or wires are inserted at random positions.

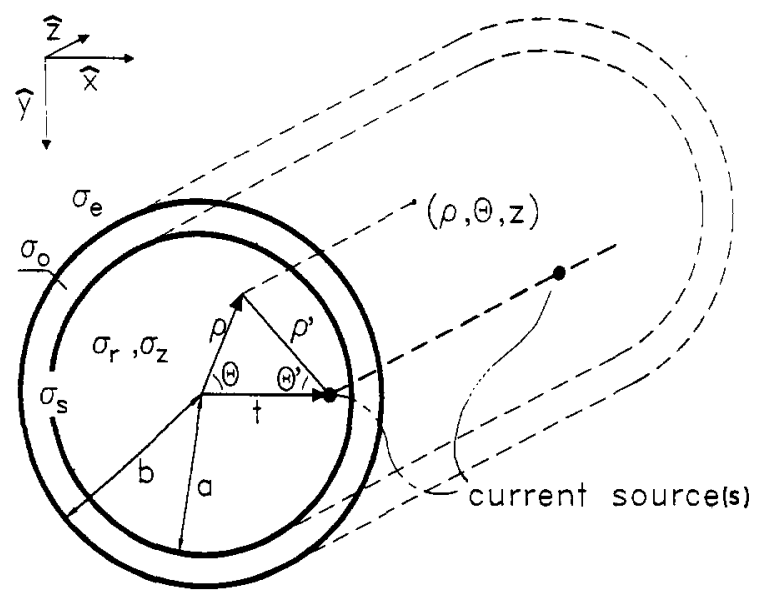

Fig. 2. The volume conduction model of the nerve and its surroundings. Longitudinal and radial conductivity inside the fascicle are $\sigma_{z}$ and $\sigma_{r}$, respectively. Perineural sheath conductivity $\sigma_{s}$; epineural conductivity $\sigma_{o}$ and extraneural conductivity, $\sigma_{e}$. For conductivity values, see the description of Fig. 7 in the text. a sheath conductivity $\sigma_{s}$. The next layer is the perineurium, with conductivity $\sigma_{\mathrm{o}}$. At the outside of the fascicle the medium is infinitely homogeneous and isotropic, with conductivity $\sigma_{\mathbf{e}}$.

Stimulation electrodes are idealised as point current sources and may be positioned anywhere in the fascicle. Using the cylinder symmetry, an analytical expression for the potentials can be derived. The potential $V_{\mathrm{e}}$ for an electrode at $(r$, $0,0)$-injecting current $I-$ consists of the sum of a source term $V_{\mathrm{e}}^{\mathrm{s}}$ :

$V_{\mathrm{e}}^{\mathrm{S}}(x, y, z)=$
$4 \pi \sqrt{\sigma_{\mathrm{r}} \sigma_{\mathrm{z}}} \sqrt{(x-r)^{2}+y^{2}+z^{2} \sigma_{\mathrm{r}} / \sigma_{\mathrm{z}}}$

and a boundary term $V_{\mathrm{e}}^{\mathrm{b}}$ which is an expansion of Bessel functions (McNeal, 1976; Rattay, 1987; Meier et al., 1991; Meier, 1992). Similarly, $V_{\mathrm{e}}^{\mathrm{s}}(x, y, z \pm \lambda)$ follow from eq. (1). 


\subsubsection{Recording}

The same type of calculation as was previously made for the case of selective stimulation of nerve fibres in rat peroneal nerve (isotropic conductor, local approach, Rutten et al., 1991b) could be applied to the case where the device is used to sense natural activity from type I afferent fibres. These calculations would lead, for example, to an optimally selective electrode interdistance of $143 \mu \mathrm{m}$ for 250 type I afferent fibres in rat peroneal nerve.

However, this method of calculating an interdistance estimate is too simplified for recording purposes. This is due to the fact that an active fibre cannot be modelled as a local one-node point source (one active node), because in an active fibre about 20 active nodes contribute to a travelling action potential, i.e. 20 nodes inject current into the nerve tissue. Therefore, the following presents a more accurate procedure. When two (not overlapping in time) action potential trains are sensed by one electrode, the trains can be detected separately when the selectivity ratio $S$ of their amplitudes $V_{1}$ and $V_{2}$ exceeds a certain threshold, i.e. when $S \geq S_{\mathrm{th}}$, for example $S \geq 1.1$, or $S \geq 2$.

Quantitative insight into the selectivity ratio $S$ as a function of spatial and conductivity parameters may be obtained by the combined use of an electrode lead field model (using the volume conduction model as outlined in the previous section, see Fig. 2) and a probability model ('Monte Carlo' simulations). The latter means that the positions of nerve fibres and electrodes are varied through the nerve fascicle in each simulation run according to a chosen type of distribution, for example random or homogeneous, or limited to a clustered sub-bundle of fibres.

\subsection{Microfabrication of the 3D multi-electrode array}

The basic structure of the 3D multi-electrode array ('brush') is created in a sawing procedure. In one inch $\langle 111\rangle$-silicon wafers, approximately $4 \mathrm{~mm}$ thick, $600 \mu \mathrm{m}$ deep grooves are sawn, at distances of $120 \mu \mathrm{m}$ apart. The walls of silicon that result from this procedure are shortened in such a way that walls with three different heights result: 600,400 and $200 \mu \mathrm{m}$. Perpendicular to the first sawing direction, new grooves of $600 \mu \mathrm{m}$ depth are sawn, resulting in rows of silicon 'needles', with differing heights. The needles in a row have equal heights. The needles are $120 \mu \mathrm{m}$ apart, and have a size of approximately $55 \times 55 \mu \mathrm{m}$. They get their final size and shape from a reactive ion etching (RIE) process, using an $\mathrm{SF}_{6} / \mathrm{O}_{2}$ plasma.

In order to get a well-conducting contact between the final base of the device (the bottoms of the needles) and the electrode sites (the tips of the needles), a layer of nickel is deposited over the silicon structure. This is carried out by electroless plating, in order to get a homogeneous covering over the device. The composition of the deposition bath was taken from Roschen (1958).

In the final array, the needles must be electrically insulated from each other. Therefore, the silicon backplate will be etched away, but first another backbone structure must be created. The brush is placed in a suspension of small glass particles in ethanol, and the glass particles are allowed to deposit between the needles. After evaporation of the ethanol, the glass is melted so that a glass plate is formed. For the electrical insulation of the needle shafts from the surrounding tissue a layer of silicon nitride is deposited all over the device by means of PECVD.

Now the silicon backplate (still carrying the needles) can be removed by anisotropic etching. Because the silicon is $\langle 111\rangle$ oriented, the process is self-aligning and etching of the needles themselves is prevented. The superfluous nickel at the base is removed, so that the needles become electrically insulated from each other. At the needle tips, the silicon nitride is now etched away by an anisotropic RIE process using a $\mathrm{CHF}_{3}$ plasma. In an electrodeposition process (Pásztor et al., 1993), the needle tips (which are the electrode sites) are then covered with a layer of iridium oxide.

Finally, the device needs to be contacted. We are currently developing a flip-chip technique to connect the brush to (de)multiplexing circuitry, in which current sources and buffer amplifiers are also integrated. A schematic cross section of the final structure is shown in Fig. 3.

\section{RESULTS}

\subsection{Selective stimulation: recruitment of force}

One aspect of selectivity is the extent to which each electrode controls as few fibres as possible 


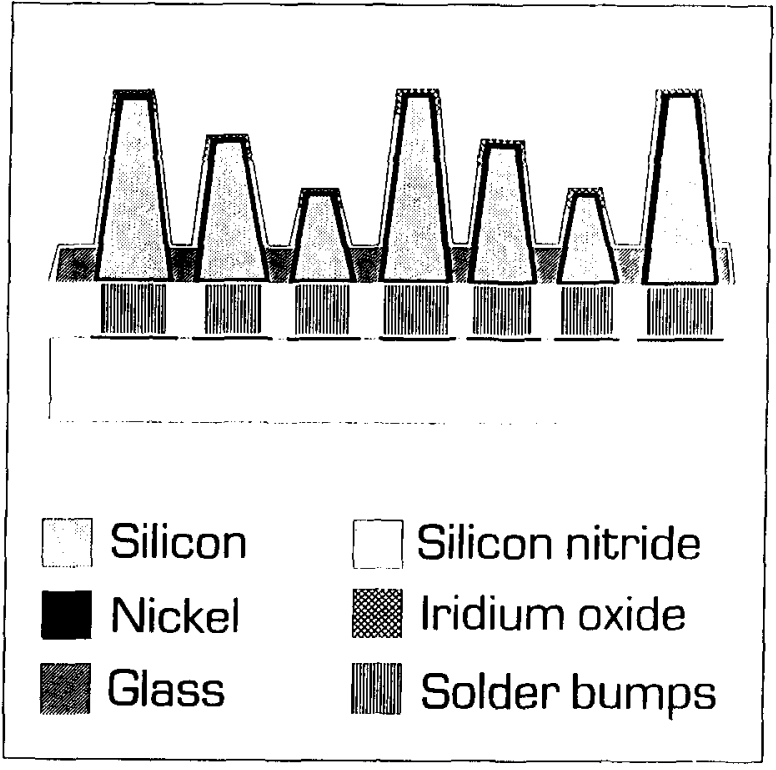

Fig. 3. Schematic cross section of the $3 D$ multi-electrode array. Processing steps are described in the text.

at low current, before potential fields start to overlap with those of other electrodes with an increase of current. This has been discussed elsewhere (Rutten et al., 1991a, b). In Rutten et al. (1991b) it was concluded that an electrode separation of $128 \mu \mathrm{m}$ was optimal for a fascicle with $350 \alpha$ motor fibres.

The other aspect is that selectivity can also be judged from the dynamic range of the recruitment curve, i.e. the more gradual the slope of this force versus current curve is, the better the control of muscle force by that electrode.

Figures 4 and 5 show recruitment curves, measured as described above (Fig. 4, force at each current level was averaged; Fig. 5, nonaveraged forces, current increased in $0.2 \mu \mathrm{A}$ steps). For comparison, the predicted slope, power three, from the local approach model has also been drawn in Fig. 4.

It can be seen that most slopes are steeper than the power three and that two curves (labelled $4 a$ and $4 b$ ) are much shallower. This has led to a more thorough and realistic type of modelling of muscle force recruitment, accompanied by an extensive set of measurements with single wire point electrodes, randomly inserted in the fascicle. The results are shown in Fig. 6 (experimental), and Fig. 7 (model simulations).

Figure 6 is a scatterplot of threshold current versus slope of a large number of force recruitment curves (10 rats), each for one position of

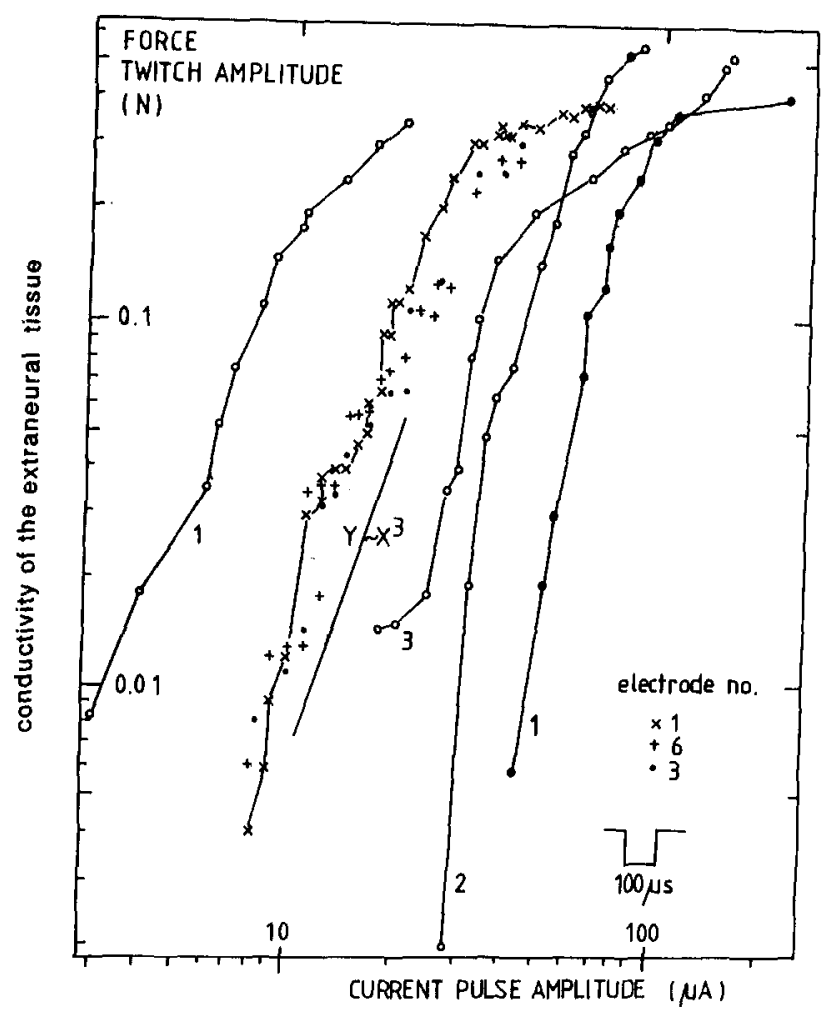

Fig. 4. Log-log recruitment curves for stimulation with the $1 D$ array. Numbers indicate electrode sites in the array. Data were taken from peroneal nerve and edl muscle in four rats. For comparison, a straight line has been drawn $\left(\mathrm{y} \sim \mathrm{x}^{3}\right)$. (See also Rutten et al., 1991a.)

a random inserted stimulating wire electrode. Threshold is defined as the current at which the force exceeds the $0.5 \mathrm{~g}$ level. The slope, $p$, is that from the straight line fit of the log-log plotted recruitment curve, according to the relationship $y$ $\sim x^{p}$ (see also Fig. 4).

Figure 6 clearly shows the effect of the outer medium conductivity: in air, (open circles), thresholds are low, $p$ extends from 2 to 12 ; in Ringer solution (filled circles), powers $p$ tend to be lower than 4 , but thresholds may vary between 5 and $20 \mu \mathrm{A}$.

As the actual number of $\alpha$-fibres to the edl muscle is only 69 (Peyronnard et al., 1986), the distribution of fibres over the nerve is rather sparse and far from homogeneous. At low currents in particular, the recruiting of the first motor units may have a very discrete and 'random' character. This is reflected in the force recruitment curves as force 'plateaus' (Meier, 1992) at low force, as well as force 'jumps'. Also, the log-log straight line fit may become dubious 


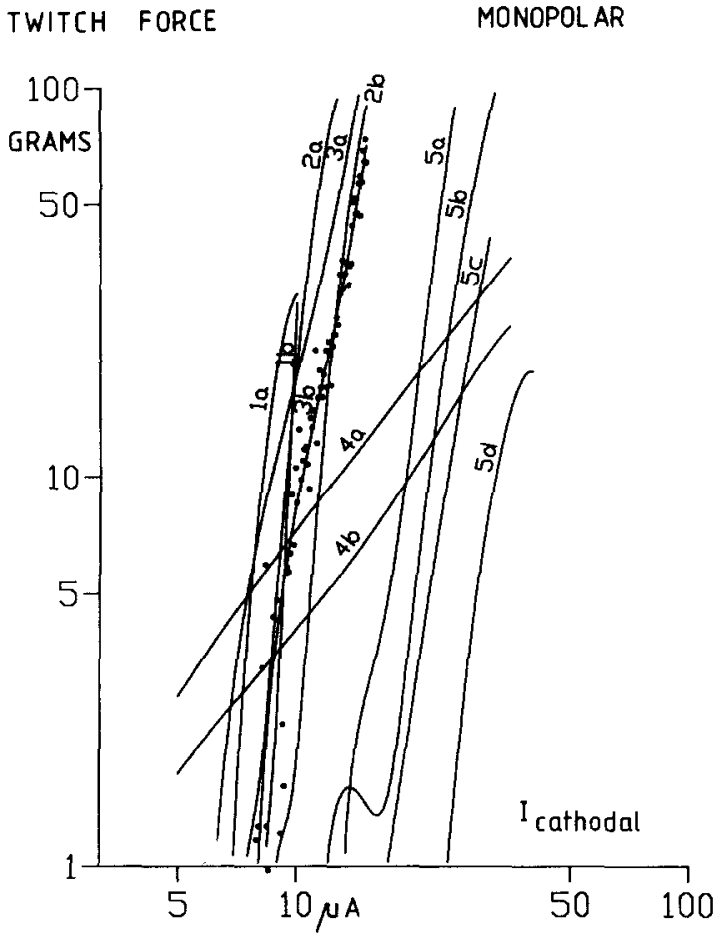

Fig. 5. Another set of recruitment curves for stimulation with the $1 D$ array (see text). Numbers indicate different animals, letters indicate different array positions. Recruitment curves have been fitted to 4th degree polynomials. (See also Meier, 1992.)

in a limited number of cases; these cases have been left out of the correlation analysis (not presented here). Also, powers $p$ may become lower as well as larger than the number three expected in a dense and homogeneous population of fibres.

Quantitatively, the same type of behaviour is seen (see Fig. 7), in the simulation model, which has realistic parameters for the diameter distribution of the 69 motor fibres, for the motor unit force distribution of the edl muscle, for the stochastic behaviour of the firing threshold and for fascicle dimensions and conductivity values (see below). However, the subdivision for the two conditions (the same separation curve has been drawn as in Fig. 6) is less obvious than in the experiments.

\subsection{Selective recording}

\subsubsection{Lead field model}

Figure 8 shows the lead field for a monopolar electrode. The horizontal axis is the axial direction of the nerve. The lead field indicates for every point in the fascicle how strong a fibre is 'seen' at the electrode when a node of the fibre is positioned at that point. Figure 8 shows the case for realistic fibres in which 20 active nodes carry the action potential. The internode distance is $1 \mathrm{~mm}$ (corresponding to a fibre with $10 \mu \mathrm{m}$ diameter) the fascicle diameter is $0.5 \mathrm{~mm}$. Figure 8 shows one elementary section of a periodic function (periodicity is $1 \mathrm{~mm}$, i.e. the internode distance). (See Meier (1992) for the volume conduction model of the nerve.) Conductivity parameters were 0.1 (radial conductivity), 0.5 (axial), $0 \cdot 1$ (epineural conductivity), $0 \cdot 1$ (extraneural) (all $1 / \Omega \mathrm{m})$ and $2000\left(1 / \Omega \mathrm{m}^{2}\right)$, (perineural sheath conductivity). Radii were $250 \mu \mathrm{m}$ (fascicle) and $270 \mu \mathrm{m}$ (nerve). Propagation velocity was $57 \mathrm{~m} / \mathrm{s}$.

Figure 8 indicates, for example, that the selectivity ratio $S$ is two for one node of one fibre at the innermost contour and another node- of a second fibre-at the next contour, which is radially $33 \mu \mathrm{m}$ away. It shows that with a discrimination limit of $S=2$, the two fibres must be separated more than $33 \mu \mathrm{m}$ radially, with the first fibre at the central contour. Conversely, two electrodes separated by a radial distance of $33 \mu \mathrm{m}$ at the most central and the second contour, would measure the signal coming from one central fibre, with an amplitude ratio of two.

\subsubsection{Probability model}

The next step is the calculation of the probability $P$ that $S$ equals or exceeds a certain threshold (e.g. $1.1,1.5$ or 2 ) for an electrode at an arbitrary place in the bundle of nerve fibres. The calculations were done in a 'Monte Carlo' way. One electrode was placed at the centre of the nerve. Forty or 100 fibres were distributed randomly over the nerve cross section, with random node position (but fixed internode distance along each fibre). This was repeated to yield 5000 realisations. For every realisation, the ratio $S$ of the two strongest action potentials (usually those nearest to the electrode), was calculated, using the lead fields. The probability $P\left(S \geq S_{\text {th }}\right)$ is the fraction of realisations for which $\mathrm{S} \geq S_{\mathrm{th}}$.

Figure 9 shows this probability as a function of the extraneural conductivity (electrode in the centre of the nerve) for selectivity thresholds $S$ $=1.1,1.5$ and 2 , with 40 active fibres. A dramatic decrease in the ability to discriminate two trains 


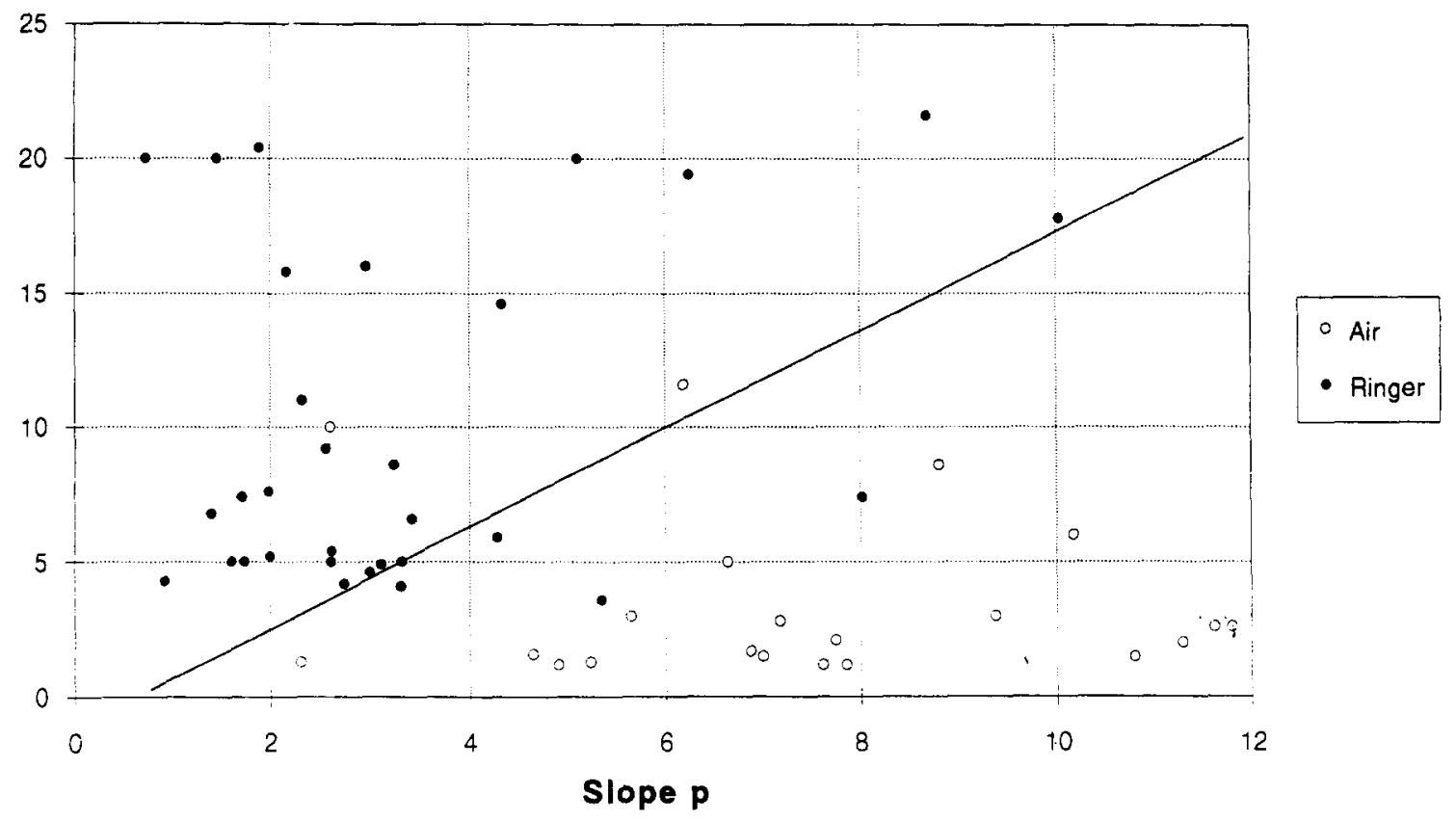

Fig. 6. Experimental threshold current versus slope $\mathrm{p}$ of 68 force recruitment curves. The nerve was either immersed in Ringer solution (41 curves) or suspended in air (27 curves). The threshold current is the amplitude of cathodal $100 \mu \mathrm{s}$ rectangular stimulation pulses for which the elicited force twitch amplitude is $0.5 \mathrm{~g}$. The slope $\mathrm{p}$ is the power of the log-log straight line fit of force versus current (see Fig. 3), $\mathrm{y} \sim \mathrm{x}^{\mathrm{p}}$, in the force interval $0.5-15 \mathrm{~g}$. The stimulating electrode was a $25 \mu \mathrm{m} \mathrm{NiCr}$ wire tip, which was randomly inserted into the fascicle. For each insertion a force recruitment curve was determined, of which the two parameters threshold and slope have been drawn in

this plot. The straight line is drawn as a guide to the eye, and separates the two conditions almost entirely.

is seen when the nerve is insulated from its surrounding tissue, i.e. for zero extraneural conductivity, e.g., when an insulating cuff is used around the nerve.

The curves saturate to the respective limiting values $S^{-3}$, which is the value that is to be expected in an analytical Poisson-model calculation (infinite space, isotropic conductivity) (Clark \& Edell, 1987; Rutten et al., 1991a).

From Monte Carlo simulations with 250 afferent fibres, it can be found that a central electrode has a volume with a radius of $40 \mu \mathrm{m}$ around it, inside which the probability is 0.99 that the fibre with the largest signal at the electrode lies in there, such that the amplitude ratio to the second largest signal (lying anywhere) $S \geq 2$. For $S \geq$ 1.1 this radius is $75 \mu \mathrm{m}$.

These outcomes suggest that multi-electrode devices should have inter-electrode distances of $80 \mu \mathrm{m}$ and $150 \mu \mathrm{m}(S \geq 2$ and $S \geq 1 \cdot 1$, respectively), and that the use of insulating cuffs around the nerve should be avoided. However, it should be noted that the calculations assume a distribution of fibres over the total cross-section of the fascicle, while in the rat fibres may be clustered in subsections of the fascicle.

\subsubsection{Calculation of single fibre responses}

The same model as presented in Section 2.2 was used to calculate the extracellular responses originating from an active fibre at the centre of the nerve. Eight positions were chosen (a-h Fig. 10) along the axial and radial dimension of the nerve. As a source, the nodal currents in 20 nodes were taken (Chiu et al., 1979). Also, the external conductivity was varied from almost insulating to well-conducting. The results (Fig. 11) show that selective recording is hampered by an insulating outer medium, implying that the use of a completely insulating cuff around a nerve should be avoided (as was also concluded in Section 3.2.2). Amplitudes in a-e are about $50 \mu \mathrm{V}$ (for the conducting outer medium), which is almost independent of the external conductivity, and the duration of the action potential is about $0.5 \mathrm{~ms}$. 


\section{Current (UA)}

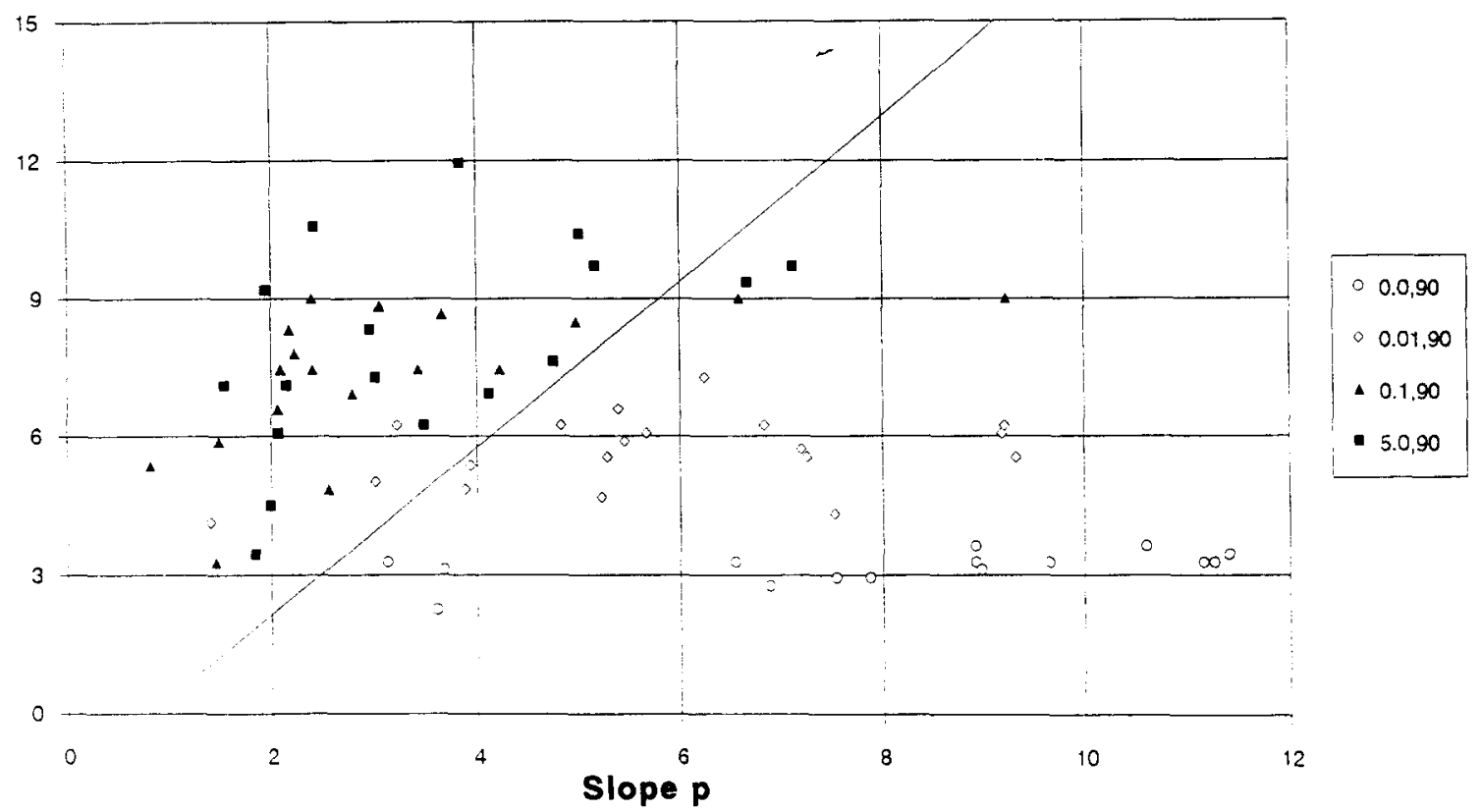

Fig. 7. The same recruitment curve parameters as in Fig. 5, but now calculated with the volume conduction model. Each simulation is for a different distribution of fibres and different conductivity parameters. Open symbols are for an insulating external medium (conductivity 0.0 and $0.01 \Omega^{-1} \mathrm{~m}^{-1}$ ), to be compared with the experimental condition 'Air'. The closed symbols are for the 'Ringer' condition (conductivity 0.1 and $5 \Omega^{-1} \mathrm{~m}^{-1}$ ). The perineurium has a conductivity of $90 \Omega^{-1} \mathrm{~m}^{-2}$. The straight line is the same as in Fig. 5 .

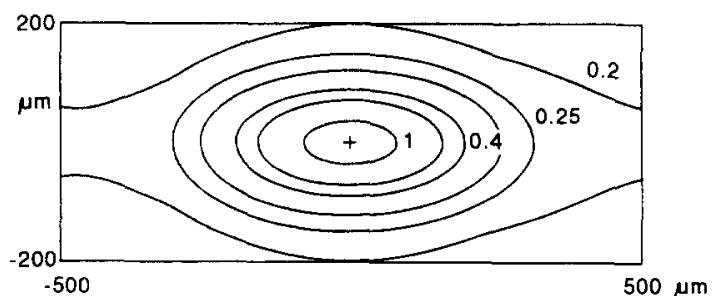

Fig. 8. Lead field equipotential contours in a crosssectional plane through the axis of the nerve (axial direction is horizortal and radial direction is vertical). The electrode is indicated by the '+' symbol. The active fibres have 20 nodes, which produce the action potential.

\subsubsection{Recordings}

Figure 12 shows some results of selective recording of afferent information in the peroneal nerve (which is exposed, but kept moist) of the rat in response to a step force mechanical stretch of the tibial muscle. A mixture of spindle and tendon responses is to be expected. Also shown is the compound ap response recorded by a bipolar hook electrode around the nerve. The figure shows this whole nerve hook response as the top trace, and also the recorded afferent activity in bipolar electrode pairs of the $1 \mathrm{D}$ array.

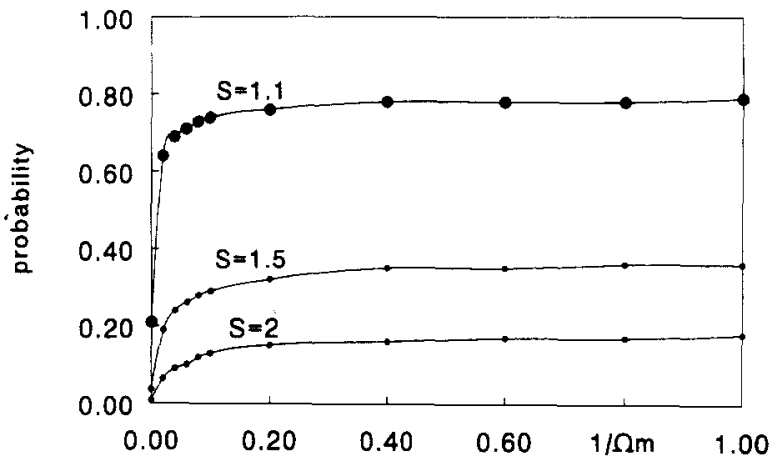

Fig. 9. The probability $\mathrm{P}\left(\mathrm{S} \geq \mathrm{S}_{t h}\right)$ that the measured action potentials from the two fibres which are nearest to a central monopolar electrode have an amplitude ratio $\mathrm{S} \geq 1 \cdot 1,1.5$ or 2 , as a function of the conductivity of the extraneural tissue. The nerve has 40 active fibres (20 nodes each).

The amplitude of the compound hook response is about $40 \mu \mathrm{V} \mathrm{pp}$, while the array responses are about $20-40 \mu \mathrm{V}$. Experiments at lower force steps show a decrease of all potentials with force, with almost the same course on all channels. 
a-1: electrodes

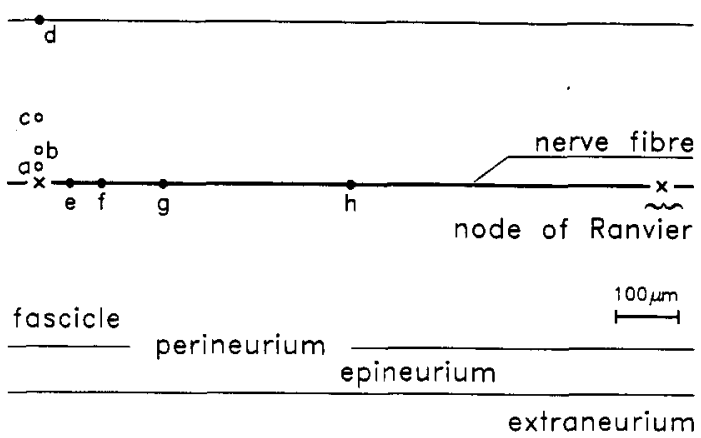

Fig. 10. Positions $a-h$ of electrodes in the nerve at which the single fibre signals of Fig. 11 have been calculated.
Another argument that these responses are from small groups (and not from single fibres) is the observed decrease with decreasing force step on all channels.

As these responses are compound responses, originating from muscle sensors which respond at different time onsets (due to the inertia of the muscle stretch, different muscle spindles and tendon organs will respond at their own threshold at different times), the exact shape of these responses cannot be deduced from the model fibre responses. Due to this complicated 'addition' of time-shifted single fibre responses, it is no surprise that the shapes are different from the ones calculated above; amplitudes and duration, are however, in the expected range.

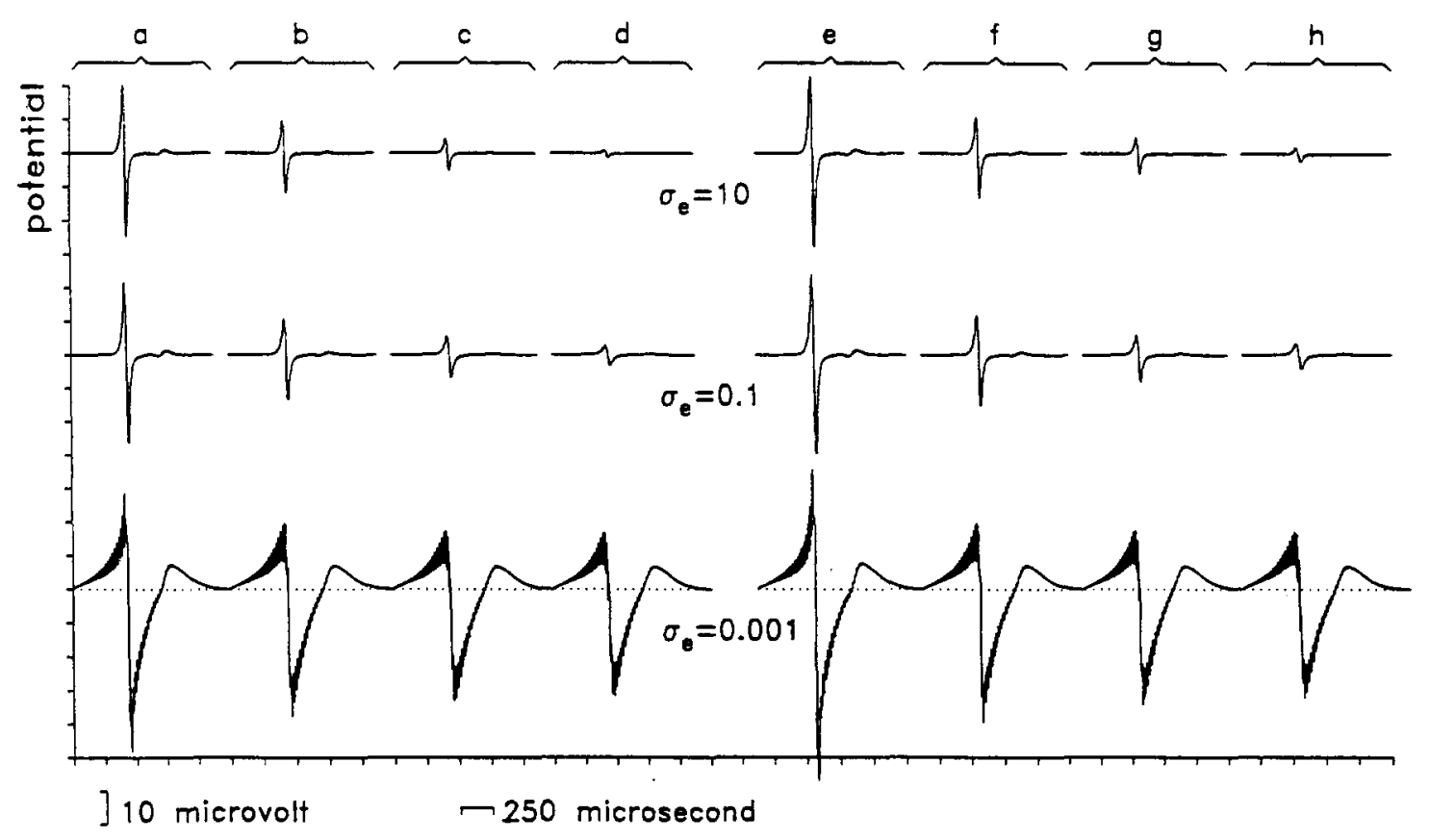

Fig. 11. Calculated single fibre signals at positions a-h (see Fig. 10), using the model of Fig. 2, for three values of the external conductivity $\sigma_{e}$, representing a well-conducting medium, intermediate or insulating. The insulating case brings selectivity $\mathrm{S}$ completely to zero.

These results can be interpreted as the compound, synchronized response of small groups of fibres; one group lies at a distance from pairs 1-2 and 3-4, almost symmetrically to both pairs, but still so close that the negative electrode number 2 in pair $1-2$ is closest. The same holds for the positive electrode number 3 in the other pair (reversed potential in 3-4 and 1-2). No fibres contribute to the pair 6-7. Another group causes the reversed signals at 8-9 and 10-12.

\subsection{D microfabrication}

Figure 13 is a schematic drawing of the completely assembled 3D multi-electrode, mounted on the electronic circuitry gate array chip. For clarity, only a portion of the $4 \times 32$ arrangement of 128 electrodes has been sketched.

The microfabrication of the 3D multi-electrode array (as described in Section 2) is currently under development. Most of the major problems 


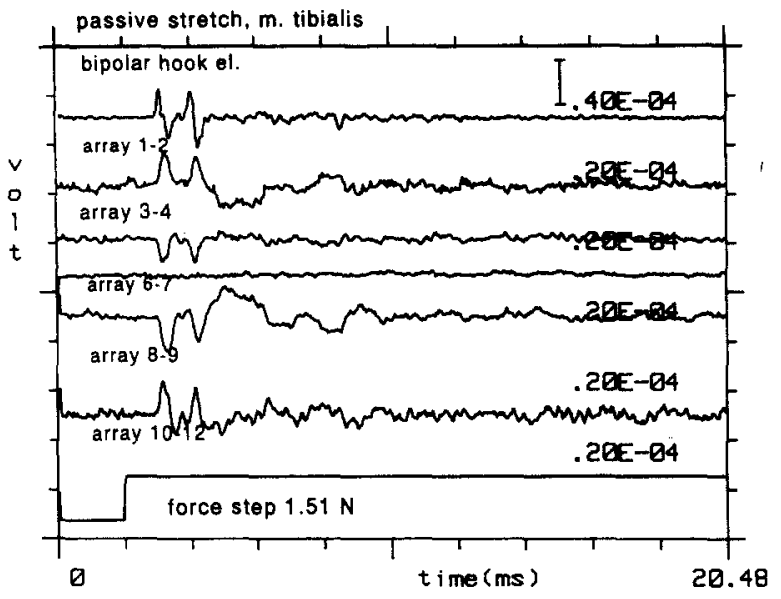

Fig. 12. Linear array bipolar recordings of afferent nerve signals in the peroneal nerve of the rat when the tibial muscle is passively stretched by a step force (bottom curve). Averaged results after 64 sweeps. The top trace shows the control compound signal, recorded with an external bipolar hook electrode in between the array and the muscle. The other traces are the bipolar recordings at electrode pairs in the linear array. For an interpretation, see text. (due to the 3D structure of the device) have been overcome. The sawing procedure and RIE steps are nicely reproducible. Figure 14 shows a scanning electron microscopy (SEM) photograph of the basic 3D 'backbone' structure of the device after sawing and subsequent 'sharpening' by RIE. Electroless nickel deposition results in a homogeneous layer of nickel all over the device, with a thickness of $1.5 \mu \mathrm{m}$. Even the problem of deposition of silicon nitride over the needle structures has been solved satisfactorily, i.e. the step coverage over the needles is good; the thickness of the nitride at the tips of the needles is $2 \mu \mathrm{m}$, and at the bottom between the needles it is approximately $1 \mu \mathrm{m}$.

After integration of all the processing steps, more extensive results will be published.

\section{DISCUSSION}

Three important determinants for graded recruitment, i.e. a large dynamic current range, are the conductivity of the external medium, the random and sparse positions of nodes and the clustering of fibres in sub-bundles in the fascicle. From the

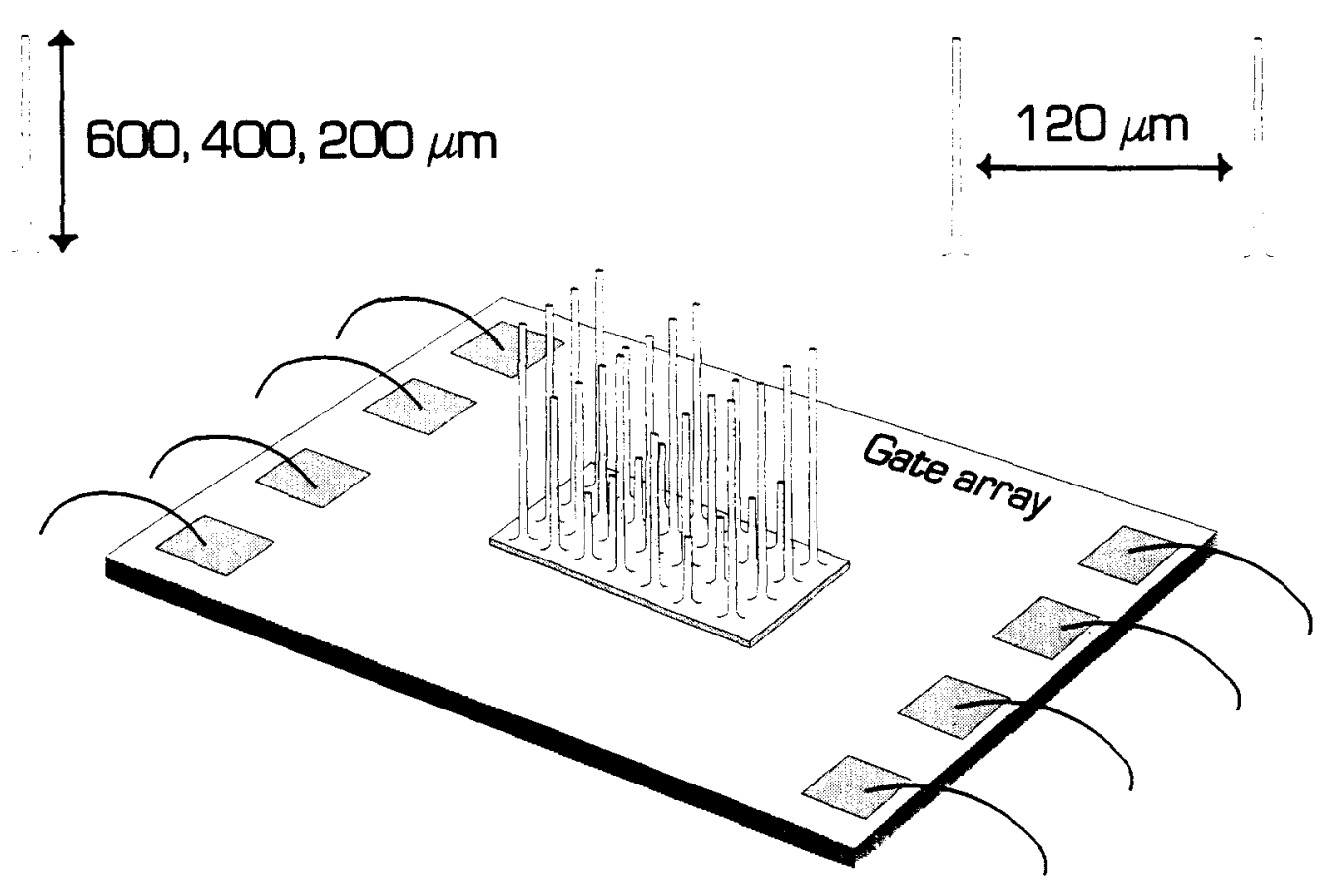

Fig. 13. Schematic drawing of the $3 D$ multi-electrode, mounted on the electronic circuitry gate array chip. For clarity, only a portion of the $4 \times 32$ arrangement of 128 electrodes has been indicated. Each needle has one iridiumoxide electrode site at its tip. 


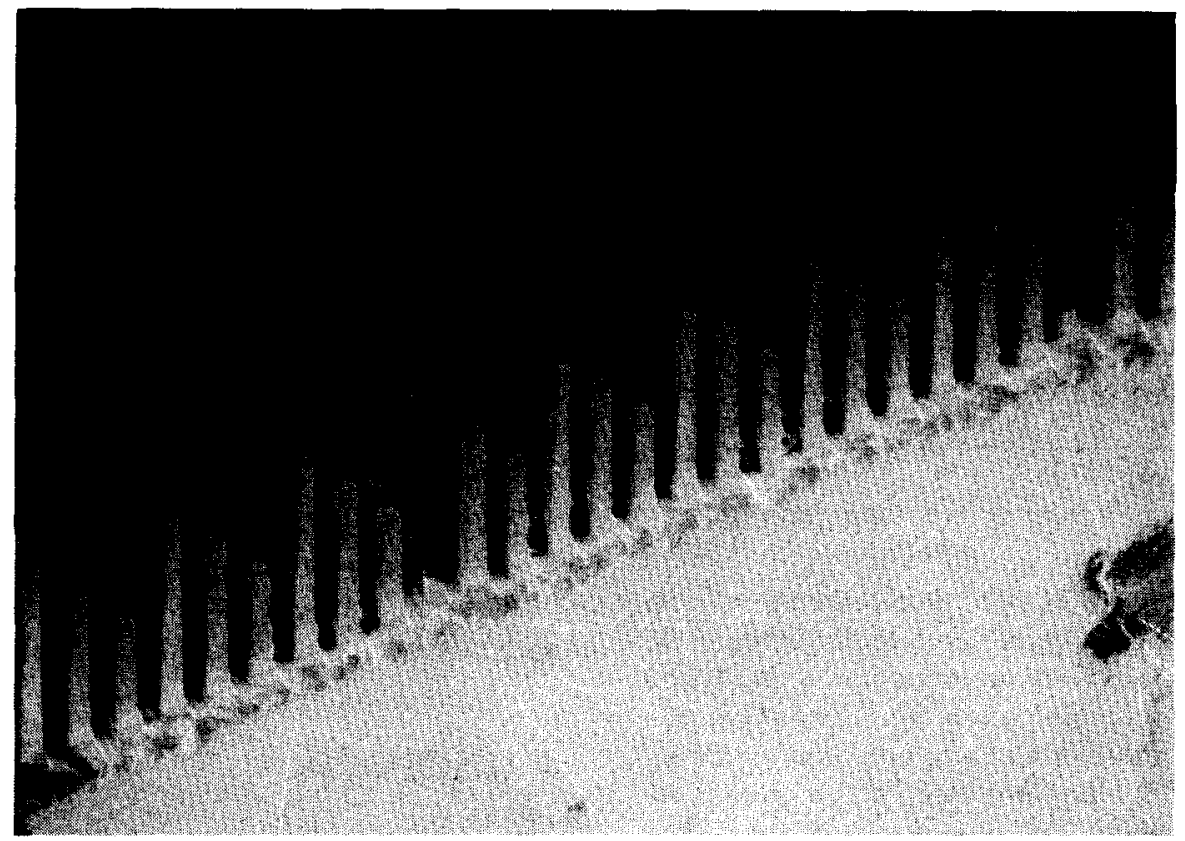

Fig. 14. Scanning electron microscopy photograph of the basic structure of the device after sawing and 'sharpening' by RIE. Note the different heights of the needle columns.

experiments and analyses in Figs. 4-7, it is clear that the largest dynamic range is obtained for nerves in Ringer solution. If nerves are embedded in an insulator, such as air, recruitment curves can be very steep, as steep as for electrodes at the skin. This is to be expected in a qualitative way, because in the insulating case the nerve will act as a conducting 'cable', with equipotential planes in the radial planes, i.e. with no voltage gradients in radial direction (see also Meier, 1992).

Further analysis (data not given) has shown that the effect of clustering of fibres in subbundles is far outweighed by the discrete and sparse character of the node positions.

We feel that the good correspondence between experiment and modelling (Figs. 6 and 7) is encouraging, but at the same time it indicates two disadvantages. First, the use of insulating cuffs (for example for mechanical support of multi-electrodes) should be avoided. Second, the sparse distribution of nodes really needs an abundance of electrodes, so that the chance of having electrodes near nodes is appreciable; otherwise, stimulating from within the nerve may be no better than from the outside of the nerve (small dynamic current range, steep recruitment curves, 'on-off' control of muscle force).
In our recordings, single fibre responses have not been detected, in spite of an extensive search during about 40 array insertions. The following may give a partial explanation. The gross calculations indicated that selectivity should be optimal for stimulation of $350 \alpha$ fibres when electrodes are separated by $128 \mu \mathrm{m}$ (or $143 \mu \mathrm{m}$ for 250 afferent fibres) (local model, homogeneous node distribution in a fixed 'lattice'). With 12 array electrodes at $50 \mu \mathrm{m}$ apart, one may expect to find single fibre responses with a substantial probability. On the other hand, the physiologicalplus-electrode noise (see Fig. 12) is about $10 \mu \mathrm{V}$ rms after averaging 64 times, which implies that single fibres can only be seen within 50-100 $\mu \mathrm{m}$ from node (radially and axially, see Figs. 10 and 11 , respectively). This decreases the probability of detecting a single fibre. Also, the bipolar way of recording reduces this distance still further: a fibre has to run close to one of the two electrodes (within 5-10 $\mu \mathrm{m}$ ), to yield enough response. (The lead field type of analysis, taking into account that more than one fibre will be active at the same time, and demanding a selectivity $S>2$, shows that a single electrode has a small 'action radius': about $40 \mu \mathrm{m}$. This is within the $50 \mu \mathrm{m}$ distance between the two differential electrodes in the linear array, so it does not imply new 
limiting conditions). It is easy to calculate that the probability of hitting a detectable single fibre action potential is not larger than $0.5-2 \%$ for one electrode pair. For an array with six pairs, this number increases to $3-12 \%$. This small probability may explain why in 40 insertions no single fibres have yet been seen. At the same time, the need for many more recording electrodes becomes very evident. The 3D multi-electrode, with 128 electrodes at $120 \mu \mathrm{m}$ apart, is a substantial step in that direction.

\section{REFERENCES}

Boyd, I.A. \& Davey, M.R. (1968). Composition of Peripheral Nerves. E. \& S. Livingstone, Edinburgh.

Brink, E.I. \& Mackel, R.G. (1993). Time course of action potentials recorded from single human afferents. Brain, 116, 415-432.

Calancie, B.M. \& Stein, R.B. (1988). Microneurography for the recording and selective stimulation of afferents: an assessment. Muscle \& Nerve, 11, 638-644.

Chiu, S.Y., Ritchie, J.M., Rogart, R.B. \& Stagg, D. (1979). A quantitative description of membrane current in rabbit myelinated nerve. J. Physiol., 292, 149-166.

Clark, L.D. \& Edell, D.J. (1987). Electrode spacing considerations for neural signal transducers. Proc. 9th IEEE/BME Conference, Boston, pp. 1660-1661.

Close, R. (1967). Properties of motor units in fast and slow skeletal muscles of the rat. J. Physiol., 193, 45-55.

Edell, D.J. (1986). A peripheral nerve information transducer for amputees: long-term multichannel recordings from rabbit peripheral nerves. IEEE Trans. Biomed. Eng., 33, 203-214.

Happak, W., Gruber, H., Holle, J., Mayr, W., Schmutterer, C., Windberger, U., Losert, U. \& Thoma, H. (1989). Multi-channel indirect stimulation reduces muscle fatigue. Proc. 11th IEEE/BME Conference, pp. 240-241.

McNeal, D. (1976). Analysis of a model for excitation of myelinated nerve. IEEE Trans. Biomed. Eng., 23, 329-337.

Meier, J.H. (1992). Selectivity and design of neuroelectronic interfaces. Thesis, University of Twente, Enschede, Netherlands.

Meier, J.H., Rutten, W.L.C., Zoutman, A.E., Boom, H.B.K. \& Bergveld, P. (1991). Simulation of multipolar fibre selective neural stimulation using intrafascicular electrodes. IEEE Trans. Biomed. Eng., 39, 122-134.

Pásztor, K., Sekiguchi, A., Shimo, N., Kitamura, N. \& Masuhara, H. (1993). Iridium oxide-based microelectrochemical transistors for $\mathrm{pH}$ sensing. Sensors \& Actuators B, 12, 225-230.

Peyronnard, J.M., Charron, L.F., Lavoie, J. \& Messier, J.P. (1986). Motor, sympathetic and sensory innervation of rat skeletal muscle. Brain Res., 373, 288-302.

Rattay, F. (1987). Ways to approximate current-distance relations for electrically stimulated fibers. J. Theor. Biol., 125, 339-349.

Robblee, L.S. \& Rose, T.L. (1990). Electrochemical guidelines for selection of protocols and electrode materials for neural stimulation. In Neural Prostheses. Fundamental Studies, Eds. W.F. Agnew and D.B. McCreery. Prentice Hall, Englewood Cliffs, NJ.

Roschen, J. (1958). Method of treating semiconductor devices. US Patent No. 2906647.

Rutten, W.L.C., van Wier, H. \& Put, J.M.H. (1991a). Sensitivity and selectivity of intraneural stimulation using a silicon electrode array. IEEE Trans. Biomed. Eng., 38, 192-198.

Rutten, W.L.C. \& Meier, J.H. (1991b). Selectivity of intraneural prosthetic interfaces for muscular control. Med. \& Biol. Eng. \& Comput., 29, NS3NS7.

Sweeney, J.D., Ksienski, D.A. \& Mortimer, J.T. (1990). A nerve cuff technique for selective excitation of peripheral nerve trunk regions. IEEE Trans. Biomed. Eng., 37, 706-715.

Swett, J.E., Wikholm, R.P., Blanks, H.I., Swett, A.L. \& Conley, L.C. (1986). Motoneurons of the rat sciatic nerve. Exp. Neurol., 93, 227-252.

Zhou, B., Baratta, R. \& Solomonow, M. (1987). Manipulation of muscle force with various firing rates and recruitment control strategies. IEEE Trans. Biomed. Eng., 34, 128-135. 